\title{
New Records for Turkish Cicindelinae (Cicindelidae) and Lebiinae (Carabidae) Fauna
}

\author{
Sema KARA ${ }^{1}$, Azize TOPER KAYGIN*2 \\ ${ }^{1}$ Bartin University, Graduate School, 74100, Bartin \\ ${ }^{2}$ Bartin University, Faculty of Forestry, Forest Entomology and Protection Department, 74100, Bartin
}

\begin{abstract}
This study includes the species of Cicindelinae and Lebiinae subfamilies belonging to the families of Cicindelidae and Carabidae collected from Bartin province between 2014 and 2016. Insect specimens belonging to these subfamilies were collected through hand picking method. At the end of the study, 1 species and 1 subspecies belonging to 1 genus of Cicindelinae subfamily (Cicindela (Cicindela) campestris Linnaeus, 1758, Cicindela (Cicindela) hybrida hybrida Linnaeus, 1758), and 2 genera and 2 species belonging to Lebiinae subfamily (Lebia (Lamprias) chlorocephala Hoffmannsegg, 1803 and Paradromius (Manodromius) linearis Olivier, 1795) were determined in Bartin province. These species were recorded as new species for Bartin's fauna. In addition, $C$. (C.) hybrida hybrida subspecies and $L$. (L.) chlorocephala species were recorded first time for Turkish fauna.
\end{abstract}

Keywords: Turkey, Bartin, Cicindelidae, Carabidae, fauna.

\section{Türkiye Cicindelinae (Cicindelidae) ve Lebiinae (Carabidae) Faunası için Yeni Kayıtlar}

\section{Öz}

$\mathrm{Bu}$ çalışma, 2014-2016 yılları arasında Bartin ilinden toplanan Cicindelidae ve Carabidae (Coleoptera) familyalarına ait Cicindelinae ile Lebiinae altfamilyalarının türlerini içermektedir. Bu altfamilyalara ait böcek örnekleri elle toplama yöntemiyle toplanmıştır. Çalışma sonucunda, Bartin ilinde Cicindelinae altfamilyasına ait 1 cinse bağlı 1 tür ve 1 alttür (Cicindela (Cicindela) campestris Linnaeus, 1758; Cicindela (Cicindela) hybrida hybrida Linnaeus, 1758) ve Lebiinae altfamilyasına ait 2 cinse bağlı 2 tür (Lebia (Lamprias) chlorocephala Hoffmannsegg, 1803; Paradromius (Manodromius) linearis Olivier, 1795) tespit edilmiştir. Bu türler Bartin faunası için yeni kayıtlardır. Buna ilave olarak, C. (C.) hybrida hybrida alttürü ile L. (L.) chlorocephala türü Türkiye faunası için ilk defa kaydedilmiştir.

Anahtar Kelimeler: Türkiye, Bartin, Cicindelidae, Carabidae, fauna. 


\section{Introduction}

Regarding both its geographical location and topographical structure, Turkey contains a wide range of species, particularly insects, and thus has the feature of a continent in terms of biological diversity. Based on this feature, it has become the center for studies by many national and foreign researchers. Moreover, Carabidae and Cicindelidae (Coleoptera) families belonging to order Coleoptera of the Insecta class have an important role considering species richness (Kesdek, 2002).

With approximately 40.000 species, Carabidae family is the third biggest family of Coleoptera after Scarabaeidae and Curculionidae families, and these species range especially in Palearctic Region (Hurka, 1996; Casale and Vigna Taglianti, 1999). Species belonging to Carabidae family can exist in almost any habitat. However, they mostly prefer humid biotopes, and some species feed as phytophagous (Zabrus spp.), some as predators (Carabus spp.) on invertebrates such as slugs and snails, and some species like Pseudoophonus rufipes De Geer as both phytophagous and predator depending on the conditions of their living environment (Lodos, 1983; Saksa and Jarosik, 2001).

Cicindelidae family members known as tiger beetles exist in many different ecological environments (from high mountains to lowlands and meadows, from tropical rainforests to deserts). Both their imagoes and larvae feed on different arthropod species as predators, live densely by streams, standing waters, ponds in particular, and move extremely fast (Thiele, 1977; Pearson, 1988; Hurka, 1996). Throughout the world, 2328 species belonging to this family have been determined. 26 of these species have been recorded in Turkey, and Cephalota eiselti (Mandl, 1967) and Homodela ismenia (Gory, 1833) species are endemic to Turkey (Cassola and Pearson, 2000; Avgin and Ozdikmen, 2007).

According to identification key developed by Chinery (1973), Carabidae and Cicindelidae are indicated as two different families (Kesdek, 2002). Most entomologists still consider these insects within their family. However, in some recent publications, tiger beetles are evaluated in Cicindelinae subfamily of Carabidae family (Jaskuła and Rewicz, 2015; Serrano and Capela, 2013; Young, 2015; Matalin and Chikatunov, 2016; Dogan Sarikaya et al., 2020). Nevertheless, this view has not been acknowledged yet by many researchers around the world, and it is claimed that, based on the distinct differences especially in their morphological structures, they need to be considered as separate families.

There are biological, ecological and faunistic studies on species of Cicindelidae and Carabidae families in Turkey's Northeastern Anatolia, Central and Eastern Black Sea regions, in provinces of Adana (Cukurova Delta), Afyon (Akdag Natural Park), Ankara, Artvin, Balıkesir (Mount Ida), Bingöl, Bursa (Uludag), Canakkale (Karabiga), Erzincan (Kemaliye), Erzurum, Eskisehir (Turkmen Mountains), Hatay (Amanos Mountains), Isparta, Izmir, Izmir (Mount Bozdag), Kahramanmaras, Kayseri, Konya, Manisa and Mugla (Aspat) (Ercelik, 1975; Turktan, 1998; Kesdek, 2002; Kocatepe, 2004; Uygun, 2005; Avgin, 2006; Karaca et al., 2006; Tezcan et al., 2006; Aydin and Kazak, 2007; Kesdek, 2007; Obali, 2007; Kocatepe and Demirsoy, 2008; Sert and Kabalak, 2010; Kocatepe, 2011; Ozturk and Kalkar, 2011; Surgut, 2011; Tanyeri, 2011; Tezcan et al., 2011; Kucukkayki, 2013; Kucukkayki et al., 2013; Avgin, 2014; Celik, 2016; Yaman, 2016; Dinler, 2019; Silay, 2019).

Different studies have been published by Kocatepe (2011), Gokturk and Celik (2017) on species of Carabidae family in Central and Eastern Black Sea regions. Similarly, in Bartin and Zonguldak provinces situated in Western Black Sea region of Turkey, studies on species of Carabidae and Cicindelidae families have been recorded by various researchers (Toper Kaygin et al., 2008; Kara and Toper Kaygin, 2018; Toper Kaygin and Kara, 2018).

\section{Material and Metot}

In the study conducted as field and laboratory research in Bartin located in the Western Black Sea region between 2014 May and 2016, 3 specimens from Cicindelinae subfamily belonging to Cicindelidae and 2 specimens from Lebiinae subfamily of Carabidae were investigated (Kara, 2016). Distribution areas of these species were recorded. Samples of the species were collected through handpicking method, killed by spraying ethyl acetate on some cotton in a glass jar, and put into collection boxes as pinned. Later, these samples were examined under microscope and identified based on the literature (Trautner and Geigenmuller, 1987; Guéorguiev and Guéorguiev, 1995; Kryzhanovskij et al., 1995; Hurka, 1996; Casale and Vigna Taglianti, 1999; Neculiseanu and Matalin, 2000; Avgin, 2006; Kocatepe, 2011; URL-1, 2015; URL-2, 2015).

\section{Results and Discussion}


Information on the species identified in Kurucasile, Amasra and Central districts of Bartin province are presented in Table 1. Cicindela (Cicindela) hybrida hybrida subspecies, Cicindela (Cicindela) campestris, Lebia (Lamprias) chlorocephala and Paradromius (Manodromius) linearis species are new records for Bartin fauna. Moreover, C. (C.) hybrida hybrida subspecies and Lebia (Lamprias) chlorocephala species are new records for Turkish fauna.

Table 1. Cicindelidae and Carabidae species and districts where they are encountered.

\begin{tabular}{lllc}
\hline Family & Species & Districts & Number \\
\hline \multirow{4}{*}{ Cicindelidae } & Cicindela (Cicindela) campestris & Kurucasile & 2 \\
& Linnaeus, 1758 & & \\
& $\begin{array}{l}\text { Cicindela (Cicindela) hybrida hybrida } \\
\text { Linnaeus 1758 }\end{array}$ & Kurucasile & 1 \\
& Lebia (Lamprias) chlorocephala & Center (Arit) & 1 \\
Carabidae & Hoffmannsegg, 1803 & Amasra (Kalesah & 1 \\
& Paradromius (Manodromius) linearis & Neighbourhood) & 1 \\
\hline
\end{tabular}

Distribution map (Figure 1), taxonomy, locality, coordinates and collected dates of identified species are given below.

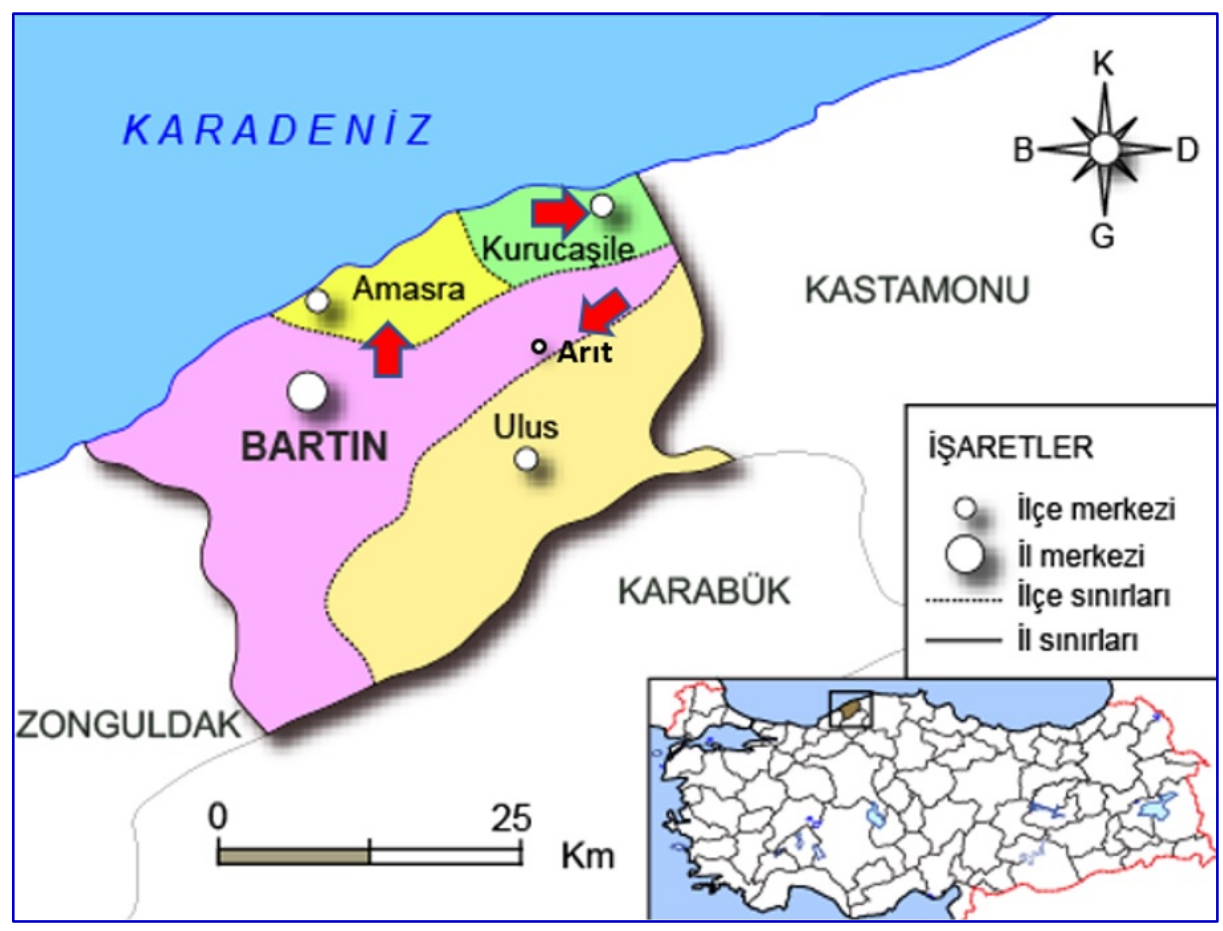

Figure 1. Location of sites sampled for Cicindelidae and Carabidae species in Bartin Province (URL-3, 2021).

\section{Family: CICINDELIDAE}

\section{Subfamily: Cicindelinae Latreille, 1802}

Genus: Cicindela Linnaeus, 1758

Species: Cicindela (Cicindela) campestris Linnaeus, 1758

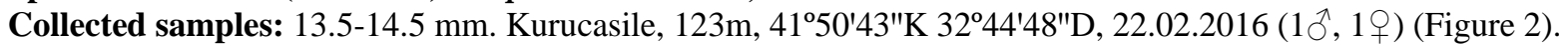

Distribution in Turkey: Zonguldak (Toper Kaygin et al., 2008), Lake Kovada National Park (Isparta) (Yaman, 2016); Aksaray, Icel (Franzen, 2007); Turkmen Mountains (Eskisehir, Kutahya) (Kucukkayki, 2013).

Worldwide distribution: Andorra, Germany, Albania, Austria, Belgium, Belarus, Bosnia Herzegovina, Bulgaria, Britain, Algeria, Czech Republic, Denmark, Estonia, Morocco, Finland, France, Georgia, Croatia, Holland, Iran, Ireland, Spain, Sweden, Switzerland, Italy, Kazakhstan, Cyprus, Kyrgyzstan, Hungary, Latvia, Lithuania, Luxemburg, Macedonia, Malta, Moldova, Norway, Uzbekistan, Poland, Portugal, Romania, Russia, Slovakia, 
Slovenia, Syria, Tunisia, Turkey, Greece (Kryzhanovskij et al., 1995; Lobl and Smetana 2003; Avgin and Ozdikmen, 2007).

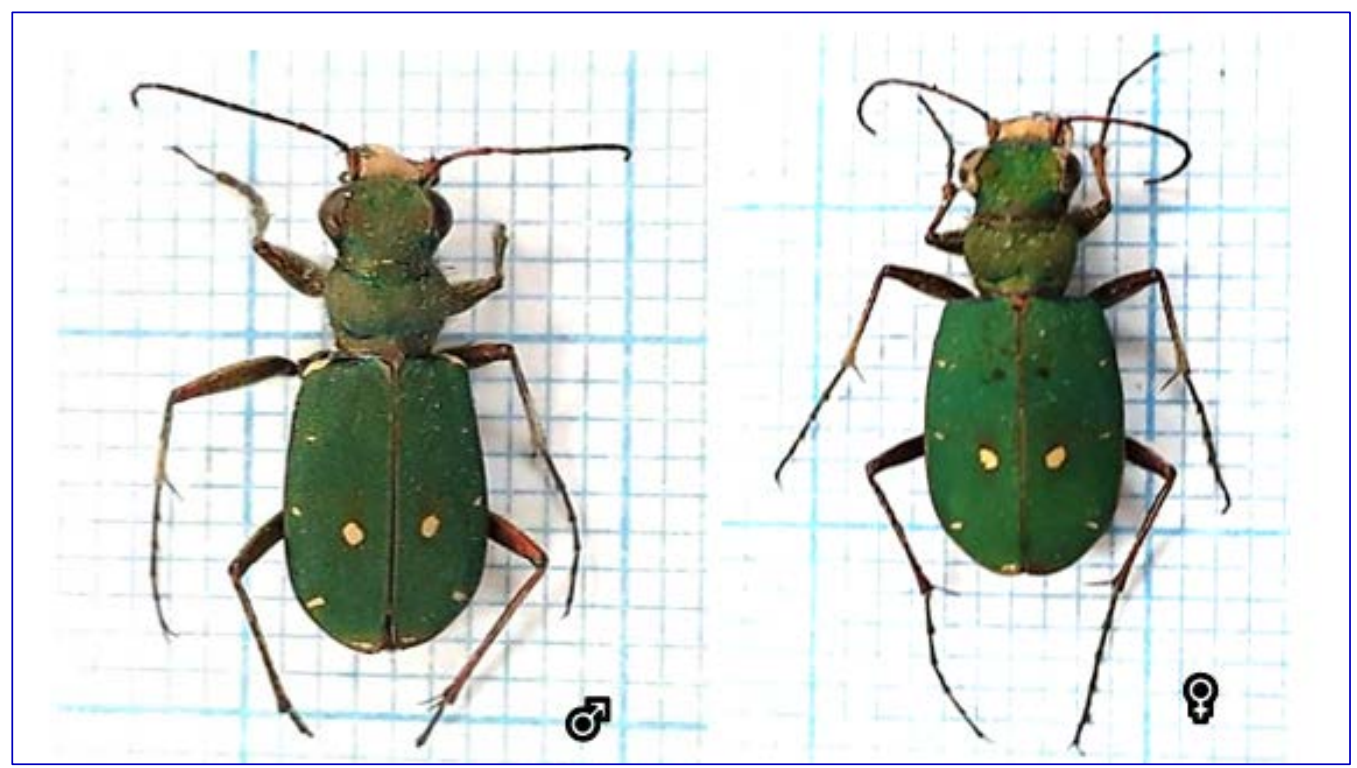

Figure 2. Cicindela (Cicindela) campestris Linnaeus, 1758.

Species: Cicindela (Cicindela) hybrida Linnaeus 1758

Subspecies: Cicindela (Cicindela) hybrida hybrida Linnaeus 1758

Collected samples: 12 mm. Kurucasile, 123m, 41 ${ }^{\circ} 50^{\prime} 43^{\prime \prime} \mathrm{K} 32^{\circ} 44^{\prime} 48^{\prime \prime} \mathrm{D}, 22.02 .2016$ (19) (Figure 3).

Worldwide distribution: Germany, Austria, Belgium, Belarus, Bosnia Herzegovina, Britain, Bulgaria, Czech Republic, Denmark, Estonia, Finland, France, Croatia, Holland, Ireland, Sweden, Kazakhstan, Latvia, Lithuania, Luxemburg, Hungary, Macedonia, Moldova, Norway, Poland, Romania, Russia, Slovakia, Slovenia, Ukraine (Kryzhanovskij et al., 1995; Jaskuła, 2005).

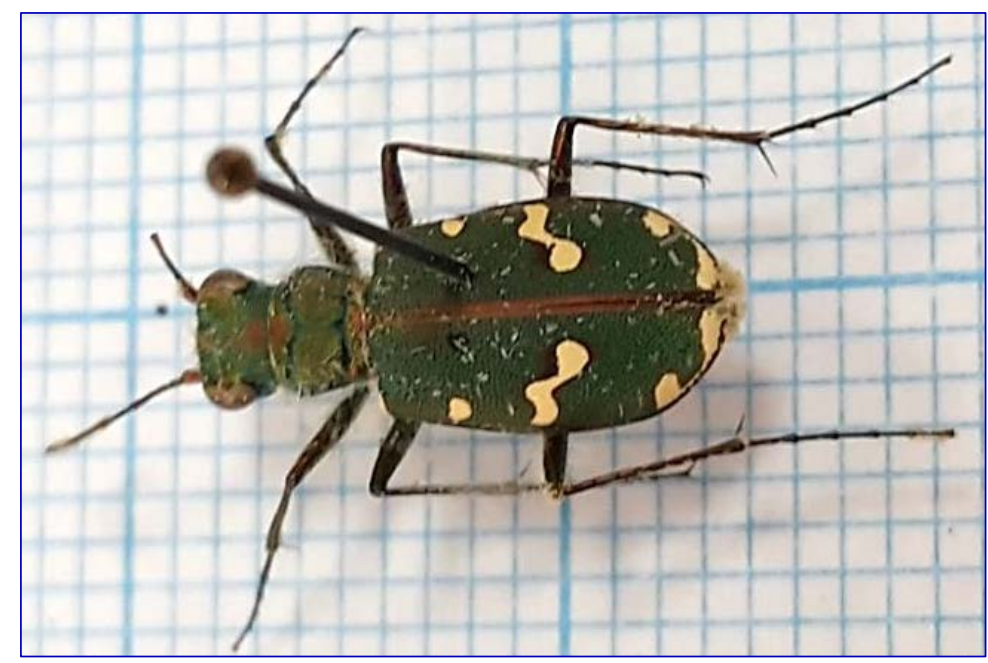

Figure 3. Cicindela (Cicindela) hybrida hybrida Linnaeus, 1758 (q).

\section{Family: CARABIDAE}

Subfamily: LEBIINAE Bonelli, 1810

Genus: Lebia Latreille, 1802

Subgenus: Lamprias Bonelli, 1810

Species: Lebia (Lamprias) chlorocephala Hoffmannsegg, 1803

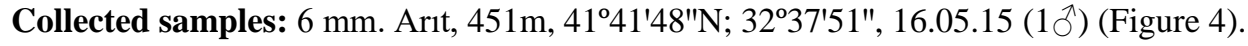


Worldwide distribution: Germany, Greece, Asia, Azerbaijan, Austria, Bosnia Herzegovina, Bulgaria, Belarus, Czech Republic, Denmark, Estonia, France, Georgia, Holland, England, Spain, Sweden, Italy, Kyrgyzstan, Kazakhstan, Latvia, Lithuania, Hungary, Moldova, Norway, Poland, Russia, Serbia, Siberia, Slovakia, Ukraine (Hurka, 1996; Chehlarov et al., 2016).

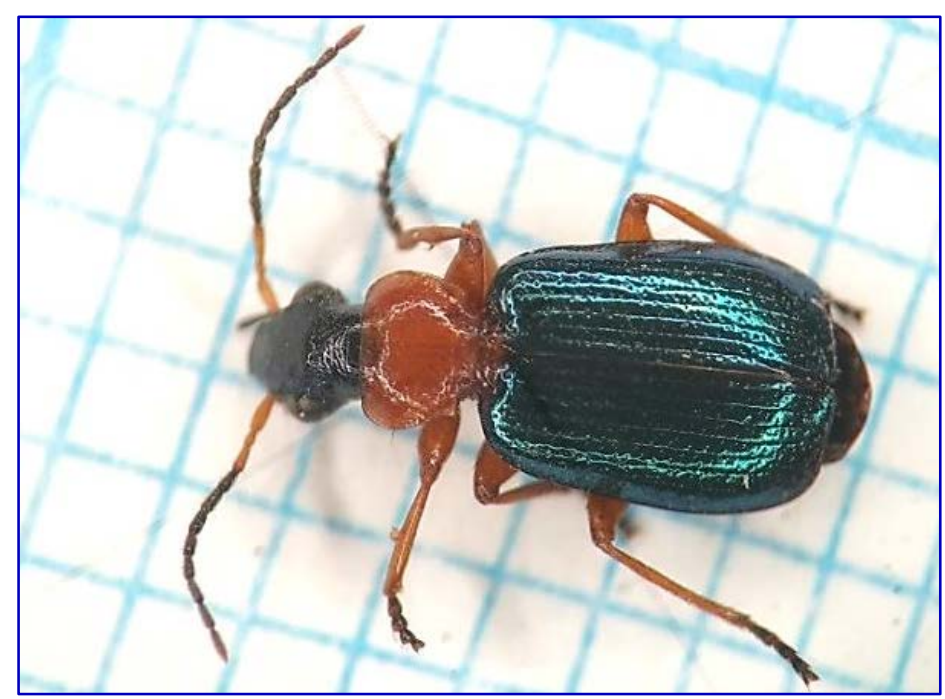

Figure 4. Lebia (Lamprias) chlorocephala Hoffmannsegg, 1803 (ð).

Genus: Paradromius Fowler, 1887

Subgenus: Manodromius Reitter, 1905

Species: Paradromius (Manodromius) linearis Olivier, 1795

Collected samples: Amasra, Kalesah neigh., 110m, 4144'24"N 32²3'49"E, 16-20.07.2014 (19) (Figure 5).

Distribution in Turkey: Anadolu (No locality record) (Casale and Vigna Taglianti, 1999).

Worldwide distribution: Russia, Ural (Hurka, 1996).

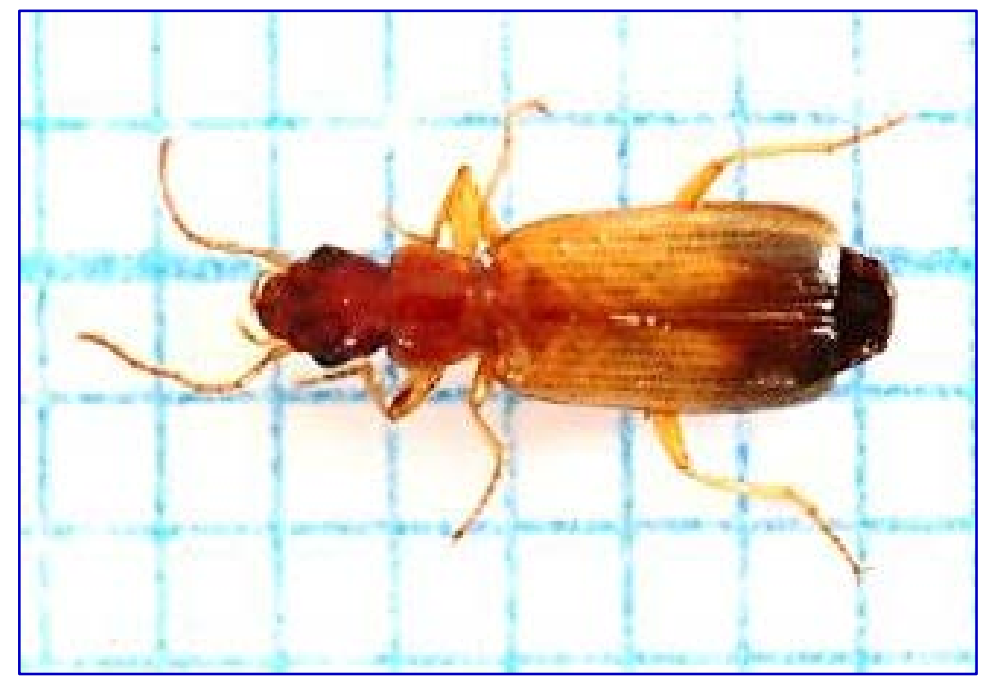

Figure 5. Paradromius (Manodromius) linearis Olivier, 1795 (†).

Franzen (2007) indicated that Cicindela (Cicindela) campestris species existed in Turkey in Aksaray (Mount Hasan) and Icel (Sertavul Pass); Yaman (2016) in Lake Kovada National Park (Isparta); and Kucukkayki (2013) in Turkmen Mountains (Eskisehir, Kutahya).

Avgin and Ozdikmen (2007), in their study on species of tiger beetles in Turkey, discovered 4 subspecies of Cicindela campestris.

Black Sea Region is divided into three as Western, Central and Eastern Black Sea (Ozcaglar, 2003; URL-4, 2021). 
In some studies, no species of Cicindelinae subfamily have been determined in Central and Eastern Black Sea regions (Kocatepe, 2011). On the other hand, in Bartin which is situated in Western Black Sea and where this study was conducted, Cicindela (Cicindela) campestris species and Cicindela (Cicindela) hybrida hybrida subspecies were identified.

Likewise, in a study on Lebiinae subfamily of Carabidae family in Central and Eastern Black Sea regions, Lebia cruxminor (Linne, 1758), Lebia cyanocephala (Linnaeus, 1758), Lebia festiva (Faldermann, 1836), Lebia trimaculata (Villers, 1789), Cymindis andreae (Ménétriés, 1832), Cymindis axillaris (Fabricius, 1794), Cymindis lineata (Quensel, 1806), Cymindis scapularis (Schaum, 1857), Cymindis variolosa (Fabricius, 1794) species were recorded (Kocatepe, 2011), yet these species were not identified in Bartin's fauna. Moreover, Lebia (Lamprias) chlorocephala (Hoffmannsegg, 1803) and Paradromius (Manodromius) linearis (Olivier, 1795) species from Lebiinae subfamily recorded in this study conducted in Bartin were not determined in other studies carried out in Central and Eastern Black Sea regions (Kocatepe, 2011). Avgin (2006) identified 14 species from Lebiinae subfamily, and L. chlorocephala and P. (M.) linearis was not included in these species.

In studies conducted in Van and its districts (Demir, 2008), Central Anatolia Region (Kocak, 2011), Karabiga area (Canakkale) (Surgut, 2011), Aspat area (Mugla) (Tanyeri, 2011), Artvin province Hatila Valley and its surrounding (Celik, 2016), Mount Madra area (Balıkesir) (Ates, 2013), and Isparta (Silay, 2019); no record of these species have been encountered.

It has been recorded with this study that $L$. (L.) chlorocephala species and C. (C.) hybrida hybrida subspecies are new records for Turkish fauna. Considering the distribution areas in the world, these determined species are Palearctic.

Local scientific studies are important as they cover comprehensive and detailed research additionally increase scientific production. Therefore, it is advised that local Cicindelidae and Carabidae fauna studies be conducted so that species diversity and richness of Turkey is more widely known.

\section{Acknowledgement}

This study was developed from Sema KARA (2016)'s master thesis titled "Bartin Carabidae (Coleoptera) Species” conducted under the supervision of Prof. Dr. Azize TOPER KAYGIN at Bartin University Institute of Sciences. Sema KARA is a PhD student financed by the Council of Higher Education's 100/2000 PhD Scholarship Program. The authors would like to express their sincere thanks to Assoc. Prof. Dr. Memiş KESDEK for the identification of insect species, valuable advice, and suggestions. They would also like to thank the anonymous reviewers for their useful comments.

\section{References}

1. Ateş, C. (2013). Madra Dağı (Balıkesir) yöresinin Carabidae, Tenebronidae, Silphidae ve Staphylinidae (Coleoptera) türlerinin çukur tuzak yöntemiyle belirlenmesi. Yüksek Lisans Tezi, Balıkesir Üniversitesi Fen Bilimleri Enstitüsü, Balıkesir, Türkiye, $120 \mathrm{~s}$.

2. Avgın, S., Ozdikmen, H. (2007). Check-list of the tiger beetles of Turkey with a review of distribution and biogeography (Coleoptera: Cicindelidae). Munis Entomology and Zoology, 2 (1): 87-102.

3. Avgın, S. S. (2006). Kahramanmaraş İli ve Çevresi Carabidae (Coleoptera) Faunası ve Taksonomisi Üzerine Çalışmalar. Doktora Tezi, Çukurova Üniversitesi, Adana, Türkiye, $352 \mathrm{s.}$

4. Avgın, S. S. (2014). Notes on the Bembidiina (Coleoptera: Carabidae: Bembidiini) from the Amanos Mountains, Turkey. The Coleopterists Bulletin, 68 (1): 131-138.

5. Aydın, G., Kazak, C. (2007). Çukurova Deltası (Adana) biyotoplarında böceklerin farklı insan aktivitelerine biyolojik gösterge olarak kullanılma olanakları. Türkiye Entomoloji Dergisi, 31 (2): 111-128.

6. Casale, A., Vigna Taglianti, A. (1999). Caraboid beetles (excl. Cicindelidae) of Anatolia, and their biogeographical significance (Coleoptera, Caraboidea). Biogeographia - The Journal of Integrative Biogeography, 20: 277-406. http://dx.doi.org/10.21426/B620110002 Retrieved from https://escholarship.org/uc/item/05p6d6sp

7. Cassola, F., Pearson, D. L. (2000). Global Patterns of tiger beetle species richness (Coleoptera: Cicindelidae): Their use in conservation planning. Biological Conservation, 95: 197-208. 
8. Chehlarov, E., Guéorguiev, B., Hristovski, S., Fancello, L., Cvetkovska-Gorgievska, A., Preliḱ, D. (2016). New Country Records and Rare and Interesting Species of Coleoptera from the Balkan Peninsula. Acta Zoologica Bulgarica 68(3):331-338.

9. Chinery, M. (1973). Insects of Britain and Northern Europe. ISBN-10: 0002120364, ISBN-13 : 9780002120364, Collins, London. 256p.

10. Celik, A. (2016). Artvin ili Hatila Vadisi ve Çevresindeki Carabidae Familyası Üzerine Çalışmalar. Yüksek Lisans Tezi, Artvin Çoruh Üniversitesi Fen Bilimleri Enstitüsü, Artvin, 110 s.

11. Demir, M. (2008). Van ve İlçelerinde Kaplanböcekleri (Cicindelidae: Coleoptera)'nın Fauna, Yayılış Alanları ve Habitat Tiplerinin Belirlenmesi. Yüksek Lisans Tezi, Yüzüncü Yıı Üniversitesi Fen Bilimleri Enstitüsü, Van, $40 \mathrm{~s}$.

12. Dinler, N. (2019). Bingöl İli Harpalinae (Coleoptera: Carabidae) Altfamilyası Üzerine Faunastik ve Sistematik Çalışmalar. Yüksek Lisans Tezi, Bingöl Üniversitesi Fen Bilimleri Enstitüsü, Bingöl, 64 s.

13. Dogan Sarikaya, A., Kocak, Y., Sarikaya, Ö. (2020). Sexual dimorphism in the Anatolian endemic tiger beetle, Cephalota circumdata ssp. cappadocica Franzen, 1996 (Coleoptera: Carabidae: Cicindelinae): a study showing the effectiveness of geometric morphometrics. Turkish Journal of Entomology, 44 (4), 425436. DOI: 10.16970/entoted.680696

14. Ercelik, M. (1975). Evolusiyon merkezi Uludağ’’n coleopter sistematiği ve bazı türlerin ekolojisi. İstanbul Üniversitesi Fen Fakültesi Monografileri.

15. Franzen, M. (2007). A new species of tiger beetle of the Cicindela campestris group from Southern Turkey, with remarks on the identity of $C$. herbacea Klug, 1832 and other taxa related to C. desertorum Dejean, 1825 (Insecta, Coleoptera, Cicindelidae). Spixiana, 30 (1): 13-24.

16. Gokturk, T., Celik, A. (2017). The family of Carabidae (Coleoptera) in Artvin Hatila National Park of Turkey. International Journal of Environment, Agriculture and Biotechnology (IJEAB), 2 (2): 2456-1878.

17. Guéorguiev, V.B., Guéorguiev, B.V. (1995). Catalogue of the ground-beetles of Bulgaria (Coleoptera: Carabidae). Pensoft Publishers, Sofia, Bulgaria.

18. Hurka, K. (1996). Carabidae of the Czech and Slovak Republics. ISBN: 9788090146624, Print Centrum. Kabourek Publisher, 565p.

19. Jaskuła, R. (2005). Mandible sexual dimorphism in Cicindela hybrida hybrida (Cicindelidae). In Protection of Coleoptera in the Baltic Sea Region. Eds. J. Skłodowski, S. Huruk, A. Barševskis, S. Tarasiuk. Warsaw Agricultural University Press, 233-239.

20. Jaskuła, R., Rewicz, T. (2015). Tiger beetles (Coleoptera: Carabidae: Cicindelinae) of Tunisia: Distribution, phenology, taxa list and new records. African Entomology. 23. 467-485. 10.4001/003.023.0217.

21. Kara, S. (2016). Bartın ili Carabidae (Coleoptera) türleri. Yayımlanmamış yüksek lisans tezi. Bartın Üniversitesi Fen Bilimleri Enstitüsü, Orman Mühendisliği Anabilim Dalı, Orman Entomolojisi ve Koruma Bilim Dalı, 165s.

22. Kara, S., Toper Kaygin, A. (2018). Carabus (Procerus) scabrosus Olivier, 1795'un Bartin İlinde Yayılış1, Biyolojisi ve Davranışları Üzerine Gözlemler. In: Keskin, N., editor, Ziraat Bilimlerinde Güncel Akademik Çalışmalar Kitabı, Cetinje-Montenegro, 153-162.

23. Karaca, İ., Karsavuran, Y., Avcı, M., Demirozer, O., Aslan, B., Sokeli, E., Bulut, H. S. (2006). Isparta ilinde Coleoptera takımına ait türler üzerinde faunistik çalışmalar. Süleyman Demirel Üniversitesi, Fen Bilimleri Enstitüsü Dergisi, 10 (2): 180-184.

24. Kesdek, M. (2002). Erzurum ili Harpalini Tribüsü (Coleoptera, Carabidae, Harpalinae) Türleri Üzerinde Faunistik ve Sistematik Çalışmalar. Yüksek Lisans Tezi, Atatürk Üniversitesi Fen Bilimleri Enstitüsü, Erzurum, $53 \mathrm{~s}$.

25. Kesdek, M. (2007). Kuzeydoğu Anadolu Bölgesi Pterostichinae (Coleoptera: Carabidae) Türleri Üzerinde Faunistik ve Sistematik Çalışmalar. Doktora Tezi, Atatürk Üniversitesi Fen Bilimleri Enstitüsü, Erzurum, Türkiye, $239 \mathrm{~s}$.

26. Kocatepe, N. (2004). Ankara İli ve İlçelerinde Carabidae (Coleoptera) Familyası Üzerinde Sistematik Çalışmalar. Yüksek Lisans Tezi, Hacettepe Üniversitesi Fen Bilimleri Enstitüsü, Ankara, Türkiye, 144 s.

27. Kocatepe, N. (2011). Orta ve Doğu Karadeniz Bölgesi Carabidae (Coleoptera) Familyası Üzerine Sistematik Çalışmalar. Doktora Tezi, Hacettepe Üniversitesi Fen Bilimleri Enstitüsü, Ankara, $196 \mathrm{~s}$.

28. Kocatepe, N., Demirsoy, A. (2008). Kemaliye (Erzincan) Carabidae (Coleoptera) Faunası. Trabzon Karadeniz Teknik Üniversitesi, 19. Ulusal Biyoloji Kongresi, 23-27 Haziran 2008; Trabzon.

29. Koçak, Y. (2011). İç Anadolu Bölgesi’nde Yayılış Gösteren Bazı Cicindelidae (Coleoptera) Türleri Üzerinde Taksonomik ve Sitogenetik Çalışmalar. Doktora Tezi. Gazi Üniversitesi Fen Bilimleri Enstitüsü, Ankara, 114s.

30. Kryzhanovskij, O. L., Belousov, I. A., Kabak, I. I., Kataev, B. M., Makarov. K. V., Shilenkov, V. G. (1995). A Checklist of the Ground Beetles of Russia and Adjacent Lands (Insecta, Coleoptera, Carabidae). ISBN-10: 9546420042, ISBN-13: 978-9546420046, Pensoft Publisher, Sofia, Bulgaria. 271p. 
31. Kucukkayki, E. (2013). Türkmen Dağları (Eskişehir-Kütahya) Yer Böcekleri (Coleoptera: Carabidae)’nin Fenolojileri ve Vertikal Tür Çeşitliliğinin Araştırılması. Yüksek Lisans Tezi, Eskişehir Osmangazi Üniversitesi Fen Bilimleri Enstitüsü, Eskişehir, $80 \mathrm{~s}$.

32. Kucukkayki, E. C., Şirin. Ü., Çalışkan, H., Şenyüz, Y. (2013). Ground beetle (Carabidae: Coleoptera) records from Kaz dagları (Ida mountain). Biological Diversity and Conservation, 6 (2): 142-149.

33. Lodos, N. (1983). Türkiye Faunasına Ait Ekin Kambur Böcekleri, Zabrus Clairville (Coleoptera: Carabidae) Cinsinin Yeniden Gözden Geçirilmesi. Türk. Bit. Kor. Derg., 7: 51-63.

34. Löbl, I., Smetana, A. (2003). Catalogue of Palaertic Coleoptera, Volume I. Archostemata-MyxophagaAdephaga. Apollo Books, Stenstrup, Denmark.

35. Matalin, A. V., Chikatunov, V. I. (2016). The tiger beetles (Coleoptera: Carabidae: Cicindelinae) of Israel and adjacent lands. ZooKeys, 578: 115-160. https://zookeys.pensoft.net/article/7383/list/18.

36. Neculiseanu, Z. Z., Matalin, A. V. (2000). A Catalogue of the ground-beetles of the Republic of Moldova (Insecta, Coleoptera: Carabidae). Pensoft Publishers, Sofia-Moskow.

37. Obalı, B. (2007). Konya İli Buğday Ekim Alanlarında Bulunan Zabrus Türleri [Zabrus spp. (Coleoptera: Carabidae)] ve Yoğunluklarının Belirlenmesi. Yüksek Lisans Tezi, Selçuk Üniversitesi Fen Bilimleri Enstitüsü, Konya, 35 s.

38. Ozcaglar, A. (2003). Türkiye'de Yapılan Bölge Ayrımları ve Bölge Planlama Üzerindeki Etkileri (The region divisions in Turkey and its effects on regional planning). https://dergipark.org.tr/tr/download/articlefile/691778. Coğrafi Bilimler Dergisi, 2003, 1 (1), 3-18.

39. Ozturk, O. O., Kalkar, O. (2011). Kahramanmaraş Menzelet Baraj Gölü çevresindeki Coleoptera faunası üzerine ön bir araştırma. KSÜ Doğa Bililimleri Dergisi, 14 (2): 22-27.

40. Pearson, D. L. (1988). Biology of tiger beetles. Annual Review Entomology, 33, 123-147.

41. Saksa, P., Jarosik, V. (2001). Laboratory studies of larval food requirements in nine species Amara (Coleoptera: Carabidae). Plant Protection Science, 37 (3): 103-100.

42. Serrano, A., Capela, R. (2013). The tiger beetles (Coleoptera: Carabidae, Cicindelinae) of Angola: a descriptive catalogue and designation of neotypes. Zootaxa, 3731(4), 401-444. doi:http://dx.doi.org/10.11646/zootaxa.3731.4.1

43. Sert, O., Kabalak, M. (2010). A study on the determination of insect fauna in Akdag Natural National Park. Hacettepe Journal of Biology and Chemistry, 38 (4): 295-305.

44. Silay, S. (2019). Isparta İli Elma Bahçelerinde Çukur Tuzak Örnekleme Yöntemi İle Yakalanan Carabidae Familyasına Ait Türler ve Biyoçeşitlilik Parametreleri. Yüksek Lisans Tezi, Isparta Uygulamalı Bilimler Üniversitesi Lisansüstü Eğitim Enstitüsü Bitki Koruma Ana Bilim Dalı, Isparta, 45s.

45. Surgut, H. (2011). Karabiga (Çanakkale) Yöresinin Carabidae, Tenebrionidae, Elateridae, Silphidae ve Staphylinidae (Coleoptera) Türlerinin Çukur Tuzak Yöntemiyle Belirlenmesi. Yüksek Lisans Tezi, Balıkesir Üniversitesi Fen Bilimleri Enstitüsü, Balıkesir, $152 \mathrm{~s}$.

46. Tanyeri, R. (2011). Aspat (Muğla) yöresi Carabidae, Tenebrionidae ve Staphylinidae (Coleoptera) Familyalarına Bağlı Türler Üzerinde Faunistik Çalışmalar. Yüksek Lisans Tezi, Ege Üniversitesi Fen Bilimleri Enstitüsü, İzmir, $70 \mathrm{~s}$.

47. Tezcan, S., Anlas, S., Jeanne, C. (2011). Species composition and habitat selection of Ground beetles (Carabidae, Coleoptera) collected by pitfall traps in Bozdaglar Mt. Western Turkey. Munis Entomology \& Zoology, 6 (2): 676-685.

48. Tezcan, S., Keskin, B., Gulpercin, N. (2006). İzmir ve Manisa İlleri Organik Kiraz Bahçelerinin Kışlak Tuzak Faunası Üzerinde Bir Değerlendirme. Uludağ Üniversitesi Türkiye 3. Organik Tarım Sempozyumu, 1 Kasım 2007, Yalova.

49. Thiele, H. U. (1977). Carabid Beetles in their Environments. Springer-Verlag, Berlin Heidelberg. New York.

50. Toper Kaygin, A., Ozkazanc, O., Kandemir, I. (2008). Insecta: Böcekler. In: Sarıbaş et al. editors. Zonguldak İli Biyoçeşitliliği. T.C. Çevre ve Orman Bakanlığ́ Zonguldak İl Müdürlüğü Doğa Koruma ve Milli Parklar Şube Müd., ISBN: 978-605-393-033-4, Bakanlık Yayın No: 362. Zonguldak, pp. 237-282.

51. Toper Kaygin, A., Kara, S. (2018). Bartin İli Carabinae ve Harpalinae (Carabidae: Coleoptera) Faunas1. In: Keskin, N., editor, Ziraat Bilimlerinde Güncel Akademik Çalışmalar Kitabı, Cetinje-Montenegro, 131151.

52. Trautner, J., Geigenmüller, K. (1987). Tiger beetles ground beetles, Illustrated key to The Cicindelidae and Carabidae of Europe. Josef Margraf Publisher, Gaimersheim.

53. Turktan, H. (1998). Eskişehir Çevresi Carabidae (Insecta: Coleoptera) Üzerine Faunistik Araştırmalar. Yüksek Lisans Tezi, Anadolu Üniversitesi Fen Bilimleri Enstitüsü Biyoloji Anabilim Dalı, Eskişehir, 34s.

54. URL-1 (2015). http://carabidae.org/, (24.05.2015).

55. URL-2 (2015). http://www.eurocarabidae.de/, (12.06.2015).

56. URL-3 (2021). http://cografyaharita.com/turkiye_mulki_idare_haritalari5.html (25.04.2021) 
57. URL-4 (2021). https://cdn-

acikogretim.istanbul.edu.tr/auzefcontent/ders/turkiyenin_ekolojik_bolgeleri/6/index.html, (17.02.2021)

58. Uygun, S. (2005). Kayseri ili Sultan Sazlığı Tabiatı Koruma Alanı İçindeki Farklı Habitatlarda Coleoptera Takımına Ait Familyalar Üzerinde Araştırmalar. Yüksek Lisans Tezi, Hacettepe Üniversitesi Fen Bilimleri Enstitüsü Biyoloji Ana Bilim Dalı, Kayseri, Türkiye, 78 s.

59. Yaman, İ. (2016). Kovada Gölü Milli Parkı (Isparta)'ndaki Yer Böcekleri (Coleoptera: Carabidae)'nin Farklı Habitatlara Bağlı Tür Çeşitliliği. Yüksek Lisans Tezi, Süleyman Demirel Üniversitesi Fen Bilimleri Enstitüsü, Biyoloji Ana Bilim Dalı, Zooloji Bilim Dalı, Isparta, 81s.

60. Young, O. P. (2015). Size relationships, early reproductive status, and mandibular wear in adult Tetracha (=Megacephala) carolina (L.) (Coleoptera: Carabidae: Cicindelinae). The Coleopterists Bulletin, 69 (1): 167-173. 